groups according to ABI. The correlation between cardiovascular risk factors, $\mathrm{ABI}$ and the $\mathrm{CAD}$ severity were analysed.

Results Gensini score $(69.0 \pm 20.1)$, three vessel $(56,56 \%)$ and $B_{2} / C$ type stenotic lesion $(79,79 \%)$ was significantly higher in $\mathrm{ABI}<0.9$ group compared with control group (50.7 \pm 17.6$)$, (19, 23.8\%), (31, $38.8 \%)$.

Result Gensini score (69.0 \pm 20.1$)$, threevessel (56, 56\%) and $\mathrm{B}_{2} / \mathrm{C}$ type stenotic lesion $(79,79 \%$ ) was significantly higher in $\mathrm{ABI}<0.9$ group, Compared with control group (50.7 \pm 17.6$)$, (19, 23.8\%), (31, 38.8\%). Binary regression analysis showed that $\mathrm{ABI}$, correlated with several risk factors, was a statistically significant independent predictor for three vessel or complex $\left(B_{2} / C\right)$ stenotic lesions and odds ratio were 3.620 and 4.011 respectively. The stepwise multivariable regression analysis shown, $A B I<0.9(R=-3.018, P<0.05)$ and age $(R=1.206$, $\mathrm{P}<0.05)$ enter the gensini score regression equation.

Conclusion $\mathrm{ABI}$ is negatively correlated with the degree of coronary artery stenosis. $\mathrm{ABI} \leq 0.9$ is a good predictor of severe coronary artery disease.

\section{E0386 EVALUATION OF CORONARY FLOW VELOCITY RESERVE IN HOMOZYGOUS FAMILIAL HYPERCHOLESTEROLAEMIA BY TRANSTHORACIC DOPPLER ECHOCARDIOGRAPHY AND DUALSOURCE CT}

doi:10.1136/hrt.2010.208967.386

Yang Ya, Wang Zheng, Zhang Xiaoshan, Li Rongjuan, Ren Hongyan, Li Zhian. Department of Ultrasound Beijing Anzhen Hospital Capital Medical University

Objective Homozygous familial hypercholesterolaemia (HoFH) is a rare disorder characterised by the early onset of atherosclerosis and usually occurrs at the ostia of coronary arteries. In this study we used transthoracic Doppler echocardiography (TTDE) to evaluate the dynamic changes of coronary flow in $\mathrm{HoFH}$ patients and to detect aortic and coronary atherosclerosis by dual-source CT (DSCT).

Method $20 \mathrm{HoFH}$ patients (12 females, 8 males, mean age $13.1 \pm 5.3$ years, with a mean low density lipoprotein (LDL) cholesterol $583 \pm 113 \mathrm{mg} / \mathrm{dl}$ ) was studied as experimental group and fifteen patients ( 8 females, 7 males, mean age $15.2 \pm 6.9$ years, with a mean low density lipoprotein (LDL) cholesterol $128 \pm 71 \mathrm{mg} / \mathrm{dl}$ ) as control group by TTDE and DSCT.

Result None of the patients showed evidence of ischaemia with standard exercise testing. Though the baseline coronary flow was similar between HoFH patients and normal controls, the hyperaemic flow velocities and thus the coronary flow velocity reserve (CFVR) were significantly lower in those with HoFH (3.36 vs 1.92 respectively, $\mathrm{p}<0.0001)$. All $\mathrm{HoFH}$ patients had aortic plaques, amongst which nine of them occurred at the coronary artery ostia, who had significantly higher LDL-cholesterol and lower CFVR than those without ostia plaques.

Conclusion Our data demonstrated that TTDE together with DSCT could be a useful non-invasive method for detection of coronary flow dynamics and atherosclerosis specifically in HoFH subjects with coronary ostia involved.

\section{e0387 PERCUTANEOUSLY INTERVENTION WAS SUCCESSFULLY PERFORMED IN RIGHT CORONARY ARTERY COMBINED WITH RIGHT SINUS OF VALSALVA ANEURYSM}

doi:10.1136/hrt.2010.208967.387

${ }^{1}$ Liu Fan, ${ }^{1}$ Lu Jingchao, ${ }^{2} \mathrm{Cui}$ Wei, ${ }^{2} \mathrm{Gu}$ Guogiang, ${ }^{2}$ Yang Xiuchun, ${ }^{2} \mathrm{Xie}$ Ruiqin, ${ }^{2} \mathrm{Hao} \mathrm{Jie}$ ${ }^{2}$ Pei Weina, ${ }^{2} \mathrm{Li}$ Baohua. ${ }^{1}$ The Second Hospital of Hebei Medical University; ${ }^{2}$ The Second Hospital of Hebei Medical University

Introduction Sinus of Valsalva aneurysms (SVA) are rare cardiac anomalies. They may be congenital or acquired and are usually asymptomatic unless complicated by such developments as intracardiac rupture with formation of a fistula or aortic valvular insufficiency. The following case is unusual in that the presenting symptom was angina pectoris due to severe atherosclerotic coronary disease with development of an aneurysm of the sinus of Valsalva secondary to atherosclerotic involvement of the aorta.

Case report A 50-year-old male patient presented with 2-week history of increasing exertional chest pain and intermittent chest pain at rest for 1 week. Echocardiography as well as contrast enhanced aorta CT revealed dilatation and irregular protrusion of the right sinus of Valsalva encroaching on right ventricular inflow tract. The aneurysm of the right sinus of Valsalva was about $4.2 \mathrm{~cm}$ in diameter. The aortic valve annulus and aortic valve leaflets were normal. Besides that, contrast enhanced aorta CT showed multiple spotted or comma calcification at aneurysm wall, and at the whole range of aorta accompany by irregular intima thickness. In addition, Selective coronary angiography showed 95\% discrete diameter stenosis at the proximal segment of right coronary artery. A $50 \%$ and a $70 \%$ tubular diameter stenosis were separately noted at the proximal segment of left descending artery and at the origin of first diagonal branch. With these findings, severe coronary atherosclerosis combined with unruptured aneurysm of the right sinus of valsalva was diagnosed. Percutaneously coronary intervention (PCI) was successfully performed, and one drug-eluted stent was deployed in the proximal right coronary artery. There was no dissection at the localisation of stent and aortic root.

Conclusions In general, the surgical method for treating severe coronary heart disease associated with unruptured SVA generally consists of coronary artery bypass grafting and closure of the opening of the aneurysm. To our knowledge, it is the first report that percutaneously intervention was successfully performed in right coronary artery combined with right sinus of valsalva aneurysm. Thereby, PCI may be an alternative treatment strategy for this clinical situation.

\section{e0388 EFFECT OF ENDOTHELIAL PROGENITOR CELLS FROM PERIPHERAL BLOOD IN PATIENTS WITH CORONARY HEART DISEASE BEFORE AND AFTER EXTRACORPOREAL CARDIAC SHOCK WAVE THERAPY}

doi:10.1136/hrt.2010.208967.388

H Y Cai, Y Wang, L Li, L Zhao, X Y Yang, J M Xiao, J M Cao, X Y Yang, C H Yang, T Guo. Department of Cardiology, First Affiliated Hospital of Kunming Medical Colledge, Kunming, China

Objective To study the effects of CSWT on the proliferation, differentiation of endothelial progenitor cells (EPCs) from peripheral blood in patients with coronary heart disease (CAD).

Methods Total mononuclear cells were isolatedfrom patients with coronary heart disease $(n=25)$ and age-matched control subjects $(n=25)$ by Ficoll density gradient centrifugation, and then the cells were plated on fibronectin coated culture dishes. After 7 days of culture. EPCs were characterised as adherent cells double positive for DiLDL-uptake and lectin binding by direct fluorescent staining under alaser scanning confocal microscope.

Results The number and proliferation of EPCs was significantly increased in patients CSWT as compared with that in control subjects $(p<0.05)$, CSWT could markedly promote the proliferation and the differentiation towards the endothelial cell lineage of EPCs in $\mathrm{CAD}$

Conclusion CSWT could markedly promote the proliferation and the differentiation towards the endothelial cell lineage of EPCs in $\mathrm{CAD}$. 